Wien klin Mag 2020 · 23:198-205 https://doi.org/10.1007/s00740-020-00353-1 Online publiziert: 10 . August 2020 (c) Der/die Autor(en) 2020

\section{Kai Markus Schneider · Christian Trautwein}

Klinik für Gastroenterologie, Stoffwechselerkrankungen und Internistische Intensivmedizin, Medizinische Klinik III, Uniklinik RWTH Aachen, Aachen, Deutschland

\title{
Die Darm-Leber-Achse bei nichtalkoholischer Fettlebererkrankung: molekulare Mechanismen und neue Targets
}

\section{Hintergrund}

Die nichtalkoholische Fettlebererkrankung (NAFLD) ist definiert als hepatische Fettakkumulation von $>5 \%$ in Abwesenheit von sekundären Faktoren, die zur Leberverfettung führen. Hierzu gehören z. B. die chronische virale Hepatitis, chronischer Alkoholabusus, autoimmune Hepatitis, kongenitale Lebererkrankungen oder die Einnahme hepatotoxischer Medikamente [1]. Die NAFLD wird als hepatische Manifestation des metabolischen Syndroms betrachtet, ist ein unabhängiger kardiovaskulärer Risikofaktor und mit weiter steigender Inzidenz mittlerweile die weltweit häufigste Lebererkrankung. Adipositas und ein vorliegender Diabetes mellitus sind die wichtigsten Risikofaktoren für die Entstehung einer NAFLD. Während die überwiegende Zahl an übergewichtigen Patienten eine NAFLD entwickelt, zeigt sich allerdings nur bei etwa einem Viertel der Patienten eine nichtalkoholische Steatohepatitis (NASH, • Abb. 1).

Die NASH stellt die entzündliche, progrediente Form der NAFLD dar und führt durch fortwährende inflammatorische Prozesse zu einer hepatozytären Schädigung. So triggert sie eine Erkrankungsprogression in Richtung Leberfibrose, -zirrhose und hepatozellulärem Karzinom. Aktuelle Forschungsarbei-

Dieser Beitrag wurde in der Zeitschrift Der Gastroenterologe 2/2020 15:112-122 https:// doi.org/10.1007/s11377-019-00402-0 erstpubliziert. Zweitpublikation mit freundlicher Genehmigung der Autoren. ten demonstrieren, dass neben genetischen Faktoren und westlicher Diät die Darm-Leber-Achse und besonders die intestinale Mikrobiota eine Schlüsselrolle für die Krankheitsprogression von NAFLD zur NASH und fortgeschrittenen Krankheitsstadien der Leberfibrose und -zirrhose spielen ([2]; • Abb. 1).

$\mathrm{Da}$ es bis heute keine zugelassene spezifische Therapie der NAFLD gibt, stellt die fortgeschrittene Leberzirrhose im Rahmen einer NAFLD mittlerweile eine der häufigsten Indikation zur Lebertransplantation dar [7]. Die stetig steigende Prävalenz, die weiterhin nicht vollständig verstandene Pathophysiologie und die bis heute fehlenden spezifischen Therapien machen die NAFLD zu einer erheblichen medizinischen Herausforderung.

\section{Bidirektionales Wechselspiel zwischen Darm und Leber bei NASH}

Forschungsarbeiten der letzten Jahre zur Biologie der Kommunikationsachse zwischen Darm und Leber haben wesentlich zum pathophysiologischen Verständnis der NAFLD beigetragen [8]. Darm und Leber kommunizieren über enge bidirektionale Verbindungen über die Gallenwege, die Pfortader und den systemischen Kreislauf miteinander. Die Leber kommuniziert mit dem Darm, indem sie Gallensäuren und bioaktive Mediatoren an die Gallenwege und den systemischen Kreislauf abgibt ([9]; • Abb. 2).

Im Darm sind diese Mediatoren aktiv und werden als endogene Substrate un- ter Mithilfe der intestinalen Mikrobiota metabolisiert. Die Galle und die darin enthaltenen Gallensäuren zirkulieren etwa 4- bis 12-mal täglich zwischen Leber und Darm und neueste Forschungsergebnisse demonstrieren, dass sie wichtige Regulationsfunktionen in der DarmLeber-Achse erfüllen [10]. Der Darm bildet die größte Grenzfläche, an der der menschliche Organismus mit der Umgebung sowie exogenen Substraten (Nahrung, Xenobiotika, Umwelteinflüsse) in Kontakt tritt. Die Leber erhält zwei Drittel ihrer Blutversorgung über die Pfortader und ist so permanent mit Produkten des mikrobiellen Metabolismus sowie mikrobiellen Bestandteilen konfrontiert. Dabei schützt sie den Wirt durch das retikuloendotheliale System vor bakterieller Translokation und systemischen Infektionen. Während Nahrungsbestandteile aufgenommen und weiterverarbeitet werden, müssen bakterielle Bestandteile oder Giftstoffe gefiltert und eine systemische bakterielle Translokation verhindert werden.

\section{Das Darmmikrobiom - Protagonist der Darm-Leber- Achse}

Alle Körperoberflächen des Menschen werden durch ein Ökosystem an Mikroorganismen aus Bakterien, Archaeen, Viren und Pilzen besiedelt. Die Gesamtheit an Mikroorganismen in einem Lebensraum wird als Mikrobiom bezeichnet [11]. Während Bakterien historisch eher im Zusammenhang mit Infektions- 


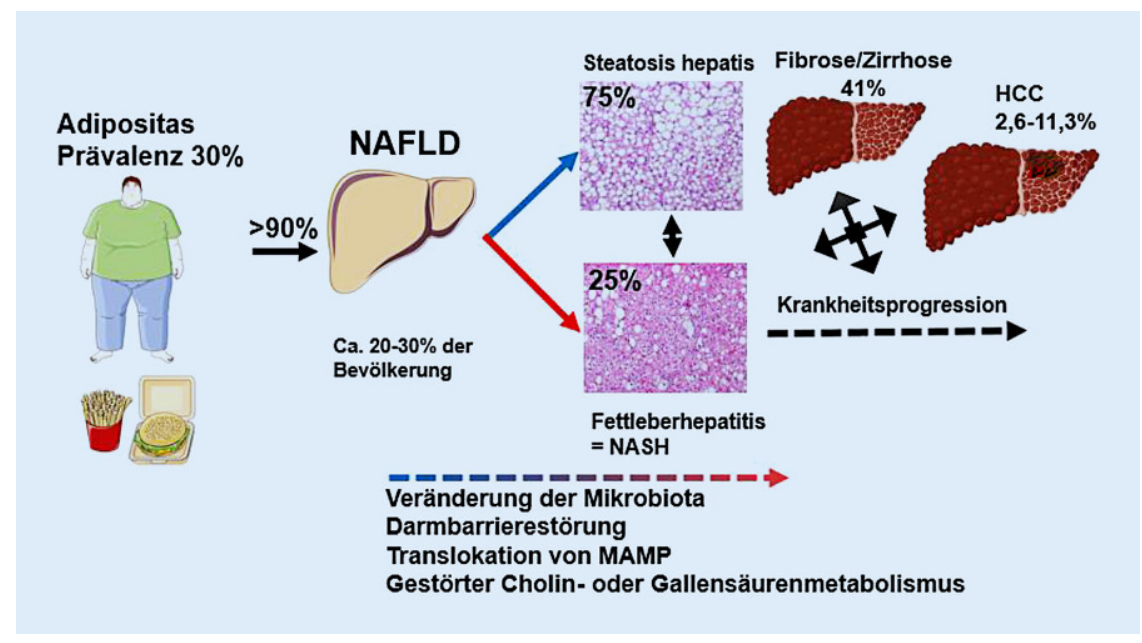

Abb. $1 \Delta$ Krankheitsprogression der nichtalkoholischen Fettlebererkrankung (NAFLD). HCC hepatozelluläres Karzinom, NASH nichtalkoholische Steatohepatitis, MAMP "microbiota associated molecular patterns". (Inzidenz Fibrose/Zirrhose bei NASH $41 \%$ aus [3]; Inzidenz HCC bei NASH 2,6-11,3\% aus [4]; Teilabbildungen aus [5], mit freundl.Genehmigung, @ Servier Medical Art by Servier, [CC by 3.0, https:// creativecommons.org/licenses/by/3.0/], Histologien aus [6], mit freundl. Genehmigung, ๑ Elsevier, alle Rechte vorbehalten)

krankheiten betrachtet wurden, ist heute bekannt, dass die unzähligen Bakterien im Darm keine untätigen Zuschauer oder gar Pathogene sind, sondern in vielfältiger Wechselwirkung miteinander stehen und aktive Akteure in diversen Prozessen der menschlichen Physiologie darstellen. Durch die strategische Position an der Grenzfläche zwischen Wirt und Umwelt fungiert das Mikrobiom als metabolisch aktives Organ und ist eine Schaltstelle der Integration von Umwelteinflüssen. So erfüllt die Mikrobiota Funktionen wie die Dekonjugation primärer Gallensäuren oder den Abbau unverdaulicher Kohlenhydrate [12]. Die personenspezifische Mikrobiotasignatur trägt im Zusammenspiel mit der Wirtsgenetik zu individuellen Merkmalen und Phänotypen bei. In Studien mit NASHPatienten wurde eine reduzierte Diversität der Mikrobiota nachgewiesen, außerdem liegt ein verändertes Verhältnis zwischen den Bakteriengruppen Bacteroidetes und Firmicutes vor [13, 14]. Auch zeigten sich Unterschiede in der generellen Mikrobiotakomposition [2]. Eine jüngere Studie zur NAFLD bei Kindern zeigte zudem eine Korrelation des NAFLD-Schweregrads mit bakteriellen Genen, die für mikrobielle Zellbestandteile („microbiota associated molecular patterns“, MAMP) kodieren [15].
In den folgenden Abschnitten werden einige spezifische Mediatoren der DarmLeber-Achse genauer diskutiert und molekulare Regelkreise aufgezeigt, die die Entstehung und das Fortschreiten von NAFLD und NASH beeinflussen.

\section{Molekulare Mechanismen und Mediatoren}

\section{Gallensäuren}

Gallensäuren sind Hauptbestandteil der Galle und erfüllen zahlreiche Funktionen, die weit über ihre Rolle bei der Bildung von Mizellen, der Verdauung und Nährstoffaufnahme hinausgehen [19]. Als Endprodukt des Cholesterinstoffwechsels werden sie in der Leber in einem mehrschrittigen enzymatischen Prozess synthetisiert und anschließend als mit Glyzin oder Taurin konjugierte primäre Gallensäuren über die kanalikuläre Membran des Hepatozyten in das Gallensystem transportiert. Über die Gallenkanälchen (Canaliculi biliferi) gelangen sie schließlich mit der Gallenflüssigkeit in das Duodenum [19]. Sobald Gallensäuren das Darmlumen erreichen, unterliegen sie dem mikrobiellen Gallensäurestoffwechsel, in dem sie zunächst dekonjugiert und anschließend enzymatisch in sekundäre Gallensäuren umgewandelt werden. Im terminalen Dünndarm werden $95 \%$ der Gallensäuren resorbiert und sie zirkulieren bis zu 6-mal täglich im enterohepatischen Kreislauf [20].

Durch die stetige Zirkulation zwischen Leber und Darm sind Gallensäuren wichtige Mediatoren, steuern spezifische Stoffwechselwege des Wirts und modulieren Entzündungsreaktionen über G-Protein-gekoppelte und nukleare Rezeptoren wie den G-Protein-gekoppelten Gallensäurerezeptor 5 (TGR5) bzw. den Farnesoidrezeptor (FXR; [10]). Durch diese immunregulatorischen Funktionen und ihre intrinsischen antimikrobiellen Eigenschaften beeinflussen Gallensäuren die Zusammensetzung der Darmmikrobiota [21]. Umgekehrt beeinflussen Veränderungen in der Zusammensetzung der Darmmikrobiota den mikrobiellen Gallensäurestoffwechsel und verändern 
wiederum die Signalübertragung über Gallensäurerezeptoren [22, 23].

Die Wechselwirkung zwischen Mikrobiota und Gallensäuren ist somit bidirektional. Zum einen werden Gallensäuren von Darmbakterien direkt enzymatisch modifiziert. Andererseits können enzymatische Modifikationen (z. B. Dekonjugation) die aktive Wiederaufnahme von Gallensäuren aus dem Dünndarm über den natriumabhängigen apikalen Gallensäuretransporter (ASBT) beeinflussen und die Signaleigenschaften von Gallensäuren modulieren [20, 24].

Im terminalen Ileum können Gallensäuren mit unterschiedlicher Rezeptoraffinität an den FXR-Rezeptor binden und dadurch die Gallensäuresynthese in der Leber beeinflussen. Eine Aktivierung des FXR-Rezeptors im Ileum führt $\mathrm{zu}$ einer verstärkten Synthese des Hormons „fibroblast growth factor 19“ (FGF 19), das anschließend über das Pfortaderblut zur Leber gelangt und dort den Fibroblast-growth-factor-Rezeptor 4 (FGFR4) aktiviert. Hierdurch wird die Expression der Cholesterol-7 $\alpha$-Monooxygenase (Cyp7A1) - des geschwindigkeitsbestimmenden Enzyms der Gallensynthese supprimiert [10]. Auch die Aktivierung von FXR in der Leber führt zu einer Suppression von Cyp7A1, wobei interessanterweise Daten daraufhindeuten, dass die FXR-Aktivierung im Darm einen stärkeren Einfluss auf den Gallensäurepool hat als in der Leber. Über den Gallensäuremetabolismus hinaus stellt der FXR-Signalweg einen zentralen Regulationsweg dar, der am Lipid- und Glukosemetabolismus beteiligt ist.

Eine Aktivierung von FXR-Agonisten zeigte in präklinischen Modellen paradoxe Effekte bei NAFLD und Fettleibigkeit $[25,26]$. Eine Defizienz des intestinalen FXR-Rezeptors sowie eine Antagonisierung von FXR schützte im Mausmodell über eine verstärkte Gallensäuresynthese vor Fettleibigkeit und Insulinresistenz [26]. Im Gegensatz dazu führte allerdings auch eine darmspezifische Aktivierung von FXR mit dem Agonisten Fexaramin zu einer Verbesserung der Insulinresistenz [25]. Diese zunächst widersprüchlich erscheinenden Ergebnisse könnten durch Unterschiede im mikrobiellen Gallensäuremetabolismus erklärt

Wien klin Mag 2020 • 23:198-205 https://doi.org/10.1007/s00740-020-00353-1

(c) Der/die Autor(en) 2020

\section{K. M. Schneider · C. Trautwein}

Die Darm-Leber-Achse bei nichtalkoholischer

Fettlebererkrankung: molekulare Mechanismen und neue Targets

\section{Zusammenfassung}

Die nichtalkoholische Fettlebererkrankung (NAFLD) ist mit weiter steigender Inzidenz die weltweit häufigste Lebererkrankung. Während Adipositas der wichtigste Risikofaktor für die Entstehung einer NAFLD ist, demonstrieren aktuelle Forschungsarbeiten, dass neben genetischen Faktoren und westlicher Diät die Darm-Leber-Achse und besonders die intestinale Mikrobiota eine Schlüsselrolle während der Krankheitsprogression spielen. Eine ungünstige
Komposition der Mikrobiota beeinflusst nicht nur den Leberstoffwechsel, sondern moduliert durch mikrobielle Moleküle und Metaboliten das inflammatorische Milieu in der Leber. Hier zeigen sich vielversprechende Regelkreise für die zukünftige Diagnostik und Therapie.

Schlüsselwörter

NASH · Mikrobiota · Bakterielle Translokation · Darm-Leber Achse · Gallensäuren

\section{The Gut-Liver Axis in Nonalcoholic Fatty Liver Disease: Molecular Mechanisms and new Targets}

\section{Abstract}

Non-alcoholic fatty liver disease (NAFLD) is the world's most common liver disease and the incidence is continuously rising. While obesity is the most important risk factor for development of NAFLD, recent data show that in addition to genetic factors and western diet, the gut-liver axis and especially the intestinal microbiota play a key role during disease progression. An unfavorable microbiota composition not only affects liver metabolism, but also modulates the inflammatory microenvironment in the liver through microbial molecules and metabolites. A better understanding of these molecular circuits may unearth promising targets for future diagnostics and therapy.

\section{Keywords}

$\mathrm{NASH} \cdot$ Microbiota $\cdot$ Bacterial translocation . Gut-liver axis · Bile acids werden [10]. Patienten mit NAFLD weisen ein deutlich verändertes Gallensäureprofil mit erhöhten Konzentrationen von primären und sekundären Gallensäuren im Serum auf [27]. Zusätzlich zeichnen sich diese Patienten durch eine herabregulierte FXR-Achse mit erhöhter CYP7A1-Aktivität und einer Vermehrung der Taurin und Glyzin metabolisierenden Bakterien aus [28].

Das Wechselspiel zwischen Mikrobiota und Gallensäuren bei NAFLD ist bisher nur unvollständig verstanden, schon jetzt offenbaren sich allerdings vielversprechende therapeutische Angriffspunkte.

\section{Mikrobielle Metaboliten}

\section{Kurzkettige Fettsäuren}

Die intestinale Mikrobiota wird basierend auf dem erstaunlichen enzymati- schen Potenzial in der Literatur zum Teil als eigenständiges metabolisch aktives Organ beschrieben [29]. Dabei nimmt die Darmmikrobiota nicht nur aktiv an der Verdauung von Nahrungsmitteln teil und ermöglicht die Aufnahme von Nahrungsbestandteilen in den portalen und systemischen Kreislauf, sondern moduliert über die Freisetzung verschiedener lokal und systemisch aktiver Metaboliten auch Stoffwechsel und Immunsystem. Mikrobielle Metaboliten haben daher sowohl lokal im Darm als auch systemisch einen fundamentalen Einfluss auf die menschliche Physiologie. In den letzten Jahren eröffnete die Entwicklung neuer Technologien zur Erforschung des Mikrobioms und mikrobieller Metaboliten ganz neue Einblicke in das komplexe Wechselspiel zwischen Wirt und Mikrobiota. Niedermolekulare mi- 


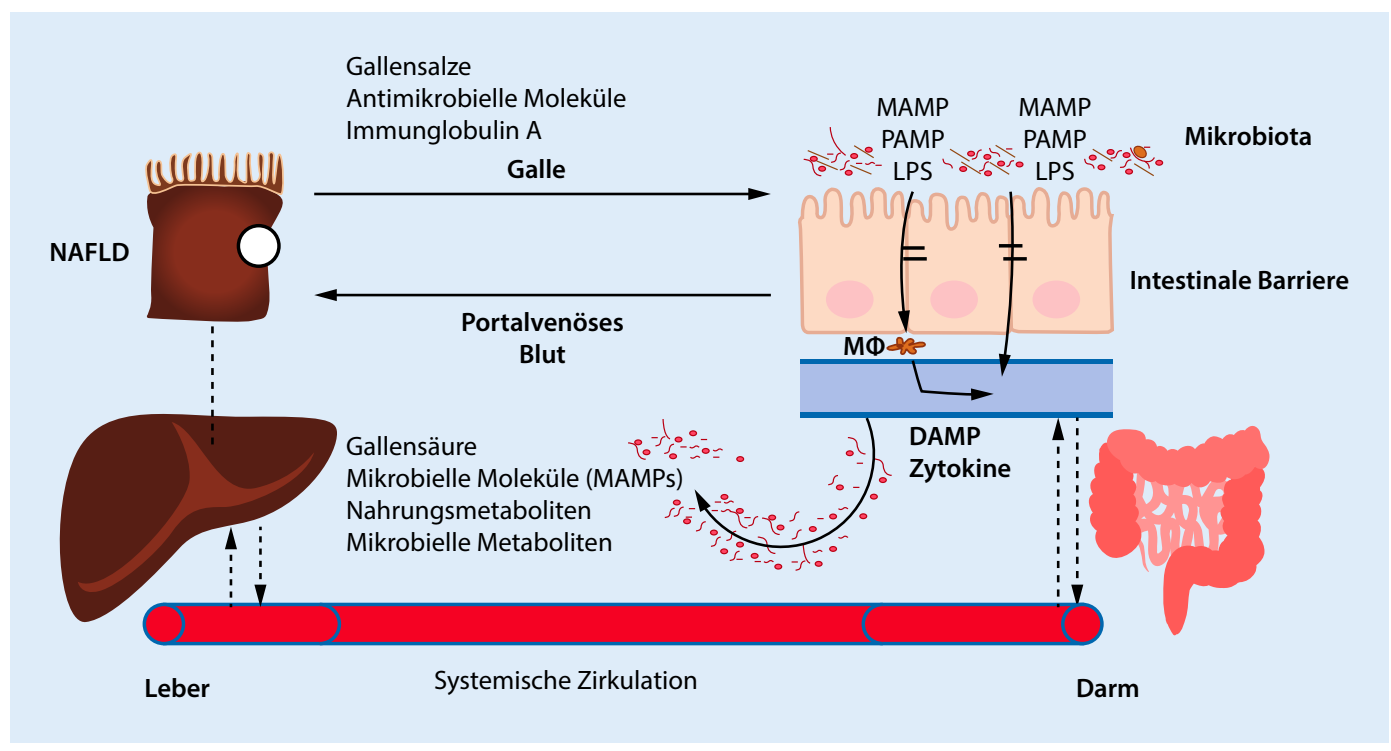

Abb. $2<$ Die Darm-Leber-Achse: Kommunikationsebenen zwischen Leber und Darm. DAMP "damageassociated molecular patterns", LPS Lipopolysaccharid, MAMP "microbiota associated molecular patterns", M $\theta$ Makrophagen, NAFLD nichtalkoholische Fettlebererkrankung, PAMP "pathogen-associated molecular patterns" krobielle Metaboliten sind dabei wichtige Mediatoren [30].

Bei fettleibigen Patienten wurde eine erhöhte Anzahl an Bakterien gefunden, die Kohlenhydrate fermentieren und so zur Biosynthese von kurzkettigen Fettsäuren („short chain fatty acids“, SCFA) beitragen [31]. Die Bildung von SCFA findet vor allem durch die Mikrobiota im Dickdarm statt. Dort dienen sie der luminalen Ernährung der Darmschleimhaut, werden aber auch über die Pfortader in die Leber transportiert und anschließend als Lipide oder Glukose gespeichert. Als Energieträger können SCFA an der Entstehung von Adipositas beteiligt sein. Sie haben aber Funktionen weit über ihre Rolle als Energieträger hinaus. So aktivieren SCFA als Signalmoleküle spezifische G-Protein-gekoppelte Rezeptoren (GPR41, GPR43 und GPR109A), regulieren über epigenetische Mechanismen (als Inhibitoren von Histondeacetylasen, HDAC) die Genexpression und steuern so Metabolismus, Immunsystem und sogar neurologische Funktionen [32]. In einem präklinischen NAFLD-Modell führte die Aktivierung von GPR43 zu einer Abnahme der Steatose [33]. Außerdem gibt es Hinweise auf eine antiinflammatorische Wirkung von SCFA. Das aktuelle Wissen über die Wirkung von SCFA bei NAFLD stammt bisher primär aus präklinischen Studien. Es bedarf weiterer klinisch-translationaler Forschung, um den Stellenwert dieser Mechanismen bei $\mathrm{Pa}$ tienten mit NAFLD zu untersuchen.
Neben SCFA konnte ein Zusammenhang eines gestörten Cholinmetabolismus mit NAFLD hergestellt werden. Als möglicher Mechanismus wird eine bakterielle Über- und Fehlbesiedlung des Dünndarms und ein damit verbundener mikrobieller Metabolismus diskutiert [34].

In präklinischen Arbeiten wurde zudem gezeigt, dass eine verminderte Konzentration bestimmter Flavonoide (sekundäre Pflanzenstoffe), die von der Mikrobiota gebildet werden, zu erneuter verstärkter Gewichtszunahme nach Diät beitragen kann. Diese wichtigen Forschungsarbeiten demonstrieren eine zentrale Rolle persistierender Mikrobiotaveränderungen bei dem vielfach beobachteten „Jo-Jo“-Effekt nach abgeschlossener Diät [35]. Mehrere Forschungsarbeiten der letzten Jahre haben zudem die endogene Produktion von Alkohol durch die Mikrobiota als einen möglichen Mechanismus der NASH diskutiert. In einer rezenten hochrangigen Publikation zeigten sich in einer chinesischen Kohorte bei NAFLD-Patienten vermehrt bestimmte alkoholproduzierende Klebsiella pneumoniae-Spezies [36]. In einem translationalen Ansatz wurde durch Invivo- und In-vitro-Experimente gezeigt, dass diese Bakterien kausal an der Entstehung der Lebererkrankung beteiligt sind. Um die Relevanz dieses Mechanismus besser einschätzen zu können, sind Untersuchungen in weiteren Kohorten notwendig.
Die endokrine Aktivität des Mikrobioms und seine Beteiligung an einer Vielzahl komplexer Krankheiten haben mikrobielle Metaboliten zu attraktiven Mediatoren neuer Therapien gemacht.

\section{Darmbarriere und bakterielle Translokation}

Neben mikrobiellen Metaboliten haben auch mikrobielle Bestandteile (MAMP) einen großen Einfluss insbesondere auf das Immunsystem des Wirts. Spezifische Rezeptoren auf Zellen vor allem des angeborenen Immunsystems („pathogen recognition receptors", PRR) können mikrobielle Moleküle (z.B. bakterielle Zellwandbestandteile wie Lipopolysaccharid, LPS) detektieren und daraufhin eine Immunreaktion aktivieren. Dies spielt sowohl lokal für das mukosale Immunsystem im Darm als auch in der Leber eine Rolle. Die Voraussetzung für eine solche Translokation bakterieller Bestandteile ist eine gestörte intestinale Barriere.

Im Zusammenhang mit dem metabolischen Syndrom kommt es häufig zu einer Störung der Darmbarriere und dadurch zur Translokation von MAMP über die Pfortader in die Leber. Dieser Mechanismus spielt nicht nur eine entscheidende Rolle für die Progression von NAFLD zur NASH, sondern ist gleichzeitig Auslöser der beim metabolischen Syndrom häufig beobachteten niedrigschwelligen systemischen 
Entzündungsreaktion. Wie neueste Forschungsarbeiten demonstrieren, scheint Hyperglykämie selbst die Funktion der intestinalen Barriere entscheidend $\mathrm{zu}$ beeinflussen [37]. Dieser Befund erklärt das erhöhte Risiko für systemische Infektionen bei schlecht eingestelltem Diabetes mellitus und hebt gleichzeitig eine gute Einstellung des Blutzuckerspiegels als Therapieziel bei Patienten mit NAFLD hervor. Es könnten so die Translokation von MAMP verhindert und konsekutive Entzündungsreaktion in der Leber reduziert werden.

In einer aktuellen Studie wurde gezeigt, dass Gallensäuren über die Aktivierung des intestinalen FXR-Rezeptors die intestinale Barriere verbessern können [38]. Die Mikrobiota-FXR-Achse ist bei Patienten mit NAFLD beeinträchtigt, sodass dieser Mechanismus auch bei Patienten zur gestörten Darmbarriere beitragen könnte [28]. Eine Therapie mit FXR-Agonisten könnte somit nicht nur einen günstigen Einfluss auf die Leber, sondern auch den Darm haben.

Eine ungünstige Mikrobiota selbst kann aber auch einen direkten Einfluss auf die Darmbarriere haben. In präklinischen Arbeiten verstärkte in einem NAFLD-Modell die intestinale Dysbiose mit Zunahme von Bakterien der Gattung Prevotella über vermehrte Aktivierung einer Toll-like-Rezeptor-vermittelten angeborenen Immunantwort die Steatohepatitis. Interessanterweise war dieser Phänotyp über den Transfer der Mikrobiota übertragbar [23]. Während eine ungünstige Mikrobiota die Krankheitsprogression antreiben kann, konnte für bestimmte bakterielle Spezies auch eine protektive Wirkung auf die Darmbarriere demonstriert werden. So kann das Bakterium Akkermansia muciniphila die Anzahl der mukusproduzierenden Becherzellen im Darm erhöhten. In einem Modell der alkoholischen Steatohepatitis führte dies zur Wiederherstellung intestinaler Tight Junctions, Verminderung der Darmdurchlässigkeit und Abnahme systemischer LPS-Spiegel. In einer aktuellen klinischen Studie wurde auch bei adipösen Patienten ein positiver Effekt auf verschiedene metabolische Parameter demonstriert [39].

\section{Diagnostische Implikationen und therapeutische Targets}

Aus dem anfänglich rein pathophysiologischen Konzept der Darm-Leber-Achse haben sich in den letzten Jahren durch präklinische Forschung konkrete therapeutische Ansätze ergeben. Basierend auf den experimentellen Daten und molekularen Mechanismen wurde eine zunehmende Zahl an klinische Studien initiiert, die therapeutisch in die Regelkreise der Darm-Leber-Achse eingreifen.

\section{Mikrobiom in der Diagnostik der $\mathrm{NASH}$}

Die frühe Diagnose von NAFLD-Patienten, die eine hohe entzündliche Aktivität und Leberfibrose aufweisen, ist von zentraler Bedeutung für die hepatologische Versorgung und stellt weiterhin eine große Herausforderung dar. In klinischen Studien wurden Assoziationen bestimmter Mikrobiotasignaturen mit dem Krankheitsstadium bei NAFLD-Patienten demonstriert. In einer aktuellen Arbeit konnten Patienten mit NALFD-Zirrhose allein basierend auf der Mikrobiotasignatur detektiert werden [13].

Insgesamt ist die Studienlage allerdings uneinheitlich und eine studienübergreifende Zirrhose-Mikrobiom-Signatur gibt es bisher nicht, sodass vor dem Einsatz in der klinischen Praxis noch weitere Grundlagenforschung erforderlich ist.

\section{Fäkaler Mikrobiomtransfer}

Die intestinale Mikrobiota weist eine erstaunliche Plastizität auf und ist grundsätzlich einer therapeutischen Modulation zugänglich. Bei therapierefraktärer pseudomembranöser Kolitis ist der fäkale Mikrobiomtransfer (FMT) ein extrem wirkungsvolles Therapieverfahren, mit dem studienübergreifend in etwa $90 \%$ der Fälle eine vollständige Krankheitsremission erzielt werden kann [40]. Die Komposition der intestinalen Mikrobiota bei Empfängern mit gutem Ansprechen zeigte nach FMT eine erhöhte mikrobielle $\alpha$-Diversität und ähnelte der Spendermikrobiota. Basierend auf diesen Befunden wurde der FMT bei verschiedenen anderen Erkrankungen mit zum Teil sehr vielversprechenden Ergebnissen erprobt. In den letzten Jahren wurde eine eindeutige Assoziation zwischen Veränderungen der Mikrobiota und NALFD hergestellt. Präklinische Daten deuten zudem darauf hin, dass die Mikrobiota eine kausale Rolle in der Krankheitsentstehung und -progression spielt [23, 41]. In mehreren klinischen Phase-I- und -II-Studien wird daher der FMT bei NAFLD und NASH erprobt (NCT02469272, NTR4339). Jüngste Daten demonstrieren, dass der FMT bei Zirrhosepatienten mit hepatischer Enzephalopathie (HE) gut vertragen wird und zu einer Verbesserung der HE sowie reduzierter Hospitalisierung führte [42].

Die Ergebnisse laufender Studien sind abzuwarten, um den Stellenwert der FMT in der Behandlung von NASH-Patienten zu bewerten. Auch gibt es weiterhin einige offene Fragen vor der Anwendung in der klinischen Routine. Es gibt zwar eine klare Assoziation zwischen Mikrobiotaveränderungen und NAFLD, allerdings ist bisher keine eindeutige studienübergreifende Mikrobiotasignatur gefunden worden. Es ist daher unklar, bei welchen Patienten die Indikation zur FMT gestellt werden sollte: Welche Patienten profitieren von dem Verfahren und kann dies basierend auf der Mikrobiotasignatur vorhergesagt werden? Wer ist der richtige Spender und gibt es eine „optimale“ Mikrobiota?

Die Mikrobiota spielt eine fundamentale Rolle in einer Vielzahl physiologischer Prozesse und interagiert mit verschiedenen Organsystemen. Dieses komplexe Wechselspiel ist bisher nur bruchstückhaft verstanden. Der Transfer einer ungünstigen Mikrobiota könnte langfristige Nebenwirkungen haben und Folgeerkrankungen hervorrufen, die bisher nicht absehbar sind. Eine gründliche Diskussion über die Indikationsstellung, eine wohl überlegte Spenderauswahl und eine gute Patientenaufklärung sind daher von entscheidender Bedeutung.

\section{Prä-, Pro- und Synbiotika}

Während der FMT auf den Austausch des gesamten mikrobiellen Ökosystems abzielt, ist auch eine direkte und möglicher- 
weise gezieltere Einflussnahme auf die Mikrobiota durch Supplementation bestimmter Nahrungsbestandteile (Präbiotika), einzelner bakterieller Spezies (Probiotika) oder einer Kombination mehrerer Mikroorganismen (z. B. Laktobazillen, E.-coli-Stämme, Bifidobakterien oder auch Hefepilze) möglich. Als Synbiotika werden Kombinationspräparate aus Präund Probiotika bezeichnet. All diese Ansätze zielen darauf ab, die Mikrobiota auf eine günstige Art zu beeinflussen, um so die intestinale Barrierefunktion zu verbessern, die Freisetzung antiinflammatorischer Metaboliten, wie SCFA durch die Mikrobiota zu fördern oder andere Stoffwechselwege (z.B. Gallensäuremetabolismus) zu beeinflussen.

Die Nahrungssupplementation mit Prä-, Pro- und Synbiotika ist in den letzten Jahren sehr populär geworden und immer wieder werden diesen Nahrungsergänzungsmitteln von der Allgemeinbevölkerung auch bei Gesunden verschiedene gesundheitsförderliche Effekte zugeschrieben. Große, gut durchgeführte klinische Studien gibt es aber nur wenige, die methodisch uneinheitlich sind und keine harten Endpunkte enthalten. Innovative Technologien der Mikrobiologie ermöglichen neue Einblicke, inwiefern Präbiotika oder Probiotika die Darmkolonisierung modulieren, die Stoffwechselaktivität der Mikrobiota beeinflussen und mit dem endogenen Mikrobiom interagieren. Nur durch sorgfältig durchgeführte Grundlagenund klinische Forschung könnten so in den nächsten Jahren das tatsächliche therapeutische Potenzial und mögliche medizinische Indikationen, aber auch Kontraindikationen dieser Ansätze herausgearbeitet werden. Auch bestehen aktuell noch viele grundlegende Fragen, die vor dem zielgerichteten Einsatz im klinischen Kontext dringend geklärt werden müssen. So ist $\mathrm{z}$. B. bis heute unklar, ob eine Kolonisierung mit probiotischen Bakterien für die gewünschte Wirkung erfolgen muss oder die Wirkung rein über bakterielle Bestandteile wie MAMP vermittelt wird. Auch ist nicht bekannt, welche Voraussetzungen durch den Wirt erfüllt werden müssen, damit es zu einer Kolonisierung kommen kann. Die molekularen Wirkmechanismen sind bisher für die meisten Probiotika nur unvollständig verstanden. Grundsätzlich kann die Wirkung über eine Veränderung des mikrobiellen Stoffwechsels und damit freigesetzter mikrobieller Metaboliten vermittelt sein, aber auch eine rein immunologische Interaktion über PRR des Wirts mit bakteriellen Zellbestandteilen ist denkbar.

Erste präklinische und klinische Studien sind durchaus vielversprechend. So führte bei übergewichtigen Patienten die Supplementation mit Akkermansia muciniphila in einer aktuellen Studie zu einer Verbesserung metabolischer Parameter. Hier zeigte sich bei pasteurisierten und damit nichtreplikativen Bakterien eine ebenso große therapeutische Wirkung wie bei lebenden Bakterien [39]. Basierend auf diesen sowie präklinischen Daten könnte eine probiotische Therapie mit Akkermansia muciniphila auch bei NASH-Patienten wirkungsvoll sein. Metaanalysen weisen auf einen positiven Effekt von Probiotika auf Surrogatparameter der NASH hin. Die Studienlage ist allerdings sehr heterogen bezüglich der verwendeten Einschlusskriterien, Probiotikaformulierungen, Behandlungsdauer und Endpunkte.

Präbiotika bestehen meist aus unverdaulichen Kohlenhydraten, zu denen verschiedene selektiv fermentierte Inhaltsstoffe, darunter Fruktane und Galaktane vom Inulintyp, zählen. Diese induzieren eine Veränderung der Komposition und Aktivität der Mikrobiota [43]. Präbiotika fördern die bakterielle Bildung von SCFA, begünstigen das Wachstum endogener Bifidobakterien und Laktobazillen, verbessern die intestinale Barrierefunktion, senken den luminalen $\mathrm{pH}$ Wert und hemmen dadurch das Wachstum von Krankheitserregern [44, 45]. In einer aktuellen Metaanalyse wurde insgesamt ein günstiger Effekt auf Surrogatparameter der NAFLD festgestellt. Die untersuchten Studien unterschieden sich methodisch jedoch erheblich und enthielten keine harten Endpunkte, sodass basierend auf diesen Daten eine abschließende Bewertung kaum möglich ist [46]. Aktuelle präklinische Daten rufen zudem zu Vorsicht vor starker Anreicherung der Diät mit fermentierbaren Ballaststoffen wie Inulin auf.
Die mikrobiotavermittelte Fermentierung des supplementierten löslichen Ballaststoffes Inulin führte in einem Mausmodell mit gestörter Mikrobiota zur Cholestase und zur Entstehung von hepatozellulären Karzinomen [47]. Diese überraschenden Daten demonstrieren, dass die Wirkung und Nebenwirkungen löslicher Ballaststoffe hochgradig kontextabhängig sind. Da Prä- und Probiotika als Nahrungsergänzungsmittel frei verkäuflich sind und gesundheitsfördernde Wirkungen als „Lifestyleprodukte“ stark beworben werden, erfolgt die Einnahme oft leichtfertig außerhalb von klinischen Studien und ohne Wissen über molekulare Wirkmechanismen und mögliche Nebenwirkungen.

Die zielgerichtete Modulation der Mikrobiota bei NAFLD birgt große Chancen, die durch sorgfältig durchgeführte Studien dank neuer Techniken der Mikrobiomforschung eröffnet werden können. Für die ungezielte Einnahme von Prä-, Pro- oder Synbiotika außerhalb von klinischen Studien gibt es bei NAFLD aufgrund eines fehlenden klaren Wirkungsnachweises und unzureichenden Verständnisses der molekularen Mechanismen bisher keine wissenschaftliche Evidenz.

\section{FXR-/FGF19-Achse und} gallensäureabhängige Signalwege

Patienten mit NAFLD zeigen erhöhte Gallensäurespiegel im Lebergewebe, Serum und auch Urin. Insbesondere zeigte sich ein erhöhtes Verhältnis eher hydrophober und zellschädigender Gallensäuren [28]. Durch ihren mikrobiellen Stoffwechsel trägt die intestinale Mikrobiota entscheidend zur Zusammensetzung des Gallensäurepools bei. Durch eine gezielte Modulation der Mikrobiota wäre es damit theoretisch möglich, den Gallensäurepool so zu verändern, dass weniger zytotoxische Gallensäuren vorliegen oder eine stärkere intestinale Aktivierung des FXR-Rezeptors erreicht wird. Ähnliche Ansätze befinden sich allerdings noch in der experimentellen präklinischen Entwicklung.

Neben einer Modulation der Mikrobiota bieten sich allerdings verschiedene direkte Angriffsmöglichkeiten in der 
FXR-/FGF19-Achse. So befinden sich aktuell verschiedene Agonisten des FXRRezeptors in der klinischen Erprobung in Phase-II- und -III-Studien. Es wurden kürzlich erste Ergebnisse von REGENERATE, einer Phase-III-Studie zu dem FXR-Agonisten Obeticholsäure bei $\mathrm{NASH}$, vorgestellt. Hier wurde der primäre Endpunkt einer Abnahme der Leberfibrose um $\geq 1$ Stufe erreicht [48]. Es zeigte sich zudem eine Verbesserung serologischer Parameter. Der therapeutische Effekt von FXR-Agonisten könnte auch über die Aktivierung intestinaler FXR-Rezeptoren und damit einer Verbesserung der Darmbarriere vermittelt werden [38]. Hier sind weitere translationale Forschungsarbeiten notwendig. Auch FGF19 ist eine attraktive Zielstruktur. Zwar zeigten frühere Studien eine Hepatokarzinogenität von FGF19 im Mausmodell, es wurde mittlerweile aber mit NGM282 eine nichttumorigene Variante von FGF19 entwickelt [49]. Dieser Wirkstoff befindet sich aktuell in der klinischen Erprobung. Erste Ergebnisse einer placebokontrollierten Phase-II-Studie bei 82 NASH-Patienten bezüglich der Wirkung auf die Steatose waren vielversprechend [50].

\section{Fazit für die Praxis}

Das aktuelle Wissen über die Rolle
der Darm-Leber-Achse bei NAFLD
fußt bisher überwiegend auf präkli-
nischen Arbeiten.
- Die Grundlagenforschung auf diesem
Gebiet hat in den letzten Jahren
interessante therapeutische Ziel-
strukturen aufgedeckt. Die genauen
molekularen Mechanismen des
Wechselspiels zwischen Mikrobiota
und Wirt innerhalb der Darm-Leber-
Achse sind allerdings weiterhin nur
unvollständig verstanden.
Entscheidend für die nächsten Jahre
sind translationale Forschungsansät-
ze, um dieses Wissen in die klinische
Anwendung zu überführen.

\section{Korrespondenzadresse}

Dr. Dr. Kai Markus Schneider

Klinik für Gastroenterologie, Stoffwechselerkrankungen und Internistische Intensivmedizin, Medizinische Klinik III, Uniklinik RWTH Aachen

Pauwelsstraße 30, 52074 Aachen, Deutschland kmschneider@ukaachen.de

Funding. Open Access funding provided by Projekt DEAL.

\section{Einhaltung ethischer Richtlinien}

Interessenkonflikt. K.M. Schneider und C. Trautwein geben an, dass kein Interessenkonflikt besteht.

Für diesen Beitrag wurden von den Autoren keine Studien an Menschen oder Tieren durchgeführt. Für die aufgeführten Studien gelten die jeweils dort angegebenen ethischen Richtlinien.

Open Access. Dieser Artikel wird unter der Creative Commons Namensnennung 4.0 International Lizenz veröffentlicht, welche die Nutzung, Vervielfältigung, Bearbeitung, Verbreitung und Wiedergabe in jeglichem Medium und Format erlaubt, sofern Sie den/die ursprünglichen Autor(en) und die Quelle ordnungsgemäß nennen, einen Link zur Creative Commons Lizenz beifügen und angeben, ob Änderungen vorgenommen wurden.

Die in diesem Artikel enthaltenen Bilder und sonstiges Drittmaterial unterliegen ebenfalls der genannten Creative Commons Lizenz, sofern sich aus der Abbildungslegende nichts anderes ergibt. Sofern das betreffende Material nicht unter der genannten Creative Commons Lizenz steht und die betreffende Handlung nicht nach gesetzlichen Vorschriften erlaubt ist, ist für die oben aufgeführten Weiterverwendungen des $\mathrm{Ma}$ terials die Einwilligung des jeweiligen Rechteinhabers einzuholen.

Weitere Details zur Lizenz entnehmen Sie bitte der Lizenzinformation auf http://creativecommons.org/ licenses/by/4.0/deed.de.

\section{Literatur}

1. Chalasani N, Younossi Z, Lavine JE, Diehl AM, Brunt EM, Cusi K, Charlton M, Sanyal AJ (2012) The diagnosis and management of non-alcoholic fatty liver disease: practice guideline by the American association for the study of liver diseases, American college of gastroenterology, and the American gastroenterological association. Hepatology. https://doi.org/10.1002/hep.25762

2. Brandl K, Schnabl B (2017) Intestinal microbiota and nonalcoholic steatohepatitis. Curr Opin Gastroenterol 33:128-133

3. Roeb E, Steffen HM, Bantel H, Baumann U, Canbay A, Demir M et al (2015) S2k Guideline non-alcoholic fatty liver disease. Z Gastroenterol 53(7):668-723

4. Yki-Jarvinen $\mathrm{H}$ (2014) Non-alcoholic fatty liver disease as a cause and a consequence of metabolic syndrome. Lancet Diabet Endocrinol 2(11):901-910

5. Servier Medical Art by Servier (2019) Webpräsenz. https://smart.servier.com/. Zugegriffen: Oktober 2019

6. Brown GT, Kleiner DE (2016) Histopathology of nonalcoholic fatty liver disease and nonalcoholic steatohepatitis. Metabolism. https://doi.org/10. 1016/j.metabol.2015.11.008

7. Tacke F, Kroy DC, Barreiros AP, Neumann UP (2016) Liver transplantation in Germany. Liver Transpl. https://doi.org/10.1002/lt.24461

8. Kolodziejczyk AA, Zheng D, Shibolet O, Elinav E (2019) The role of the microbiome in NAFLD and NASH. EMBO Mol Med. https://doi.org/10.15252/ emmm.201809302

9. Macpherson AJ, Heikenwalder M, Ganal-Vonarburg SC (2016) The liver at the nexus of host-microbial interactions. Cell Host Microbe 20:561-571

10. Schneider KM, Albers S, Trautwein C (2018) Role of bile acids in the gut-liver axis. J Hepatol 68:1083-1085. https://doi.org/10.1016/j.jhep. 2017.11.025

11. Ursell LK, Metcalf JL, Parfrey LW, Knight R (2012) Defining the human microbiome. Nutr Rev. https:// doi.org/10.1111/j.1753-4887.2012.00493.x

12. Qin J, Li R, Raes J, Arumugam M, Burgdorf KS, Manichanh C, Nielsen T, Pons N, Levenez F, Yamada T, Mende DR, Li J, Xu J, Li S, Li D, Cao J, Wang B, Liang $\mathrm{H}$, Zheng $\mathrm{H}$, Xie $\mathrm{Y}$, Tap J, Lepage $P$, Bertalan $M$, Batto JM, Hansen T, Le Paslier D, Linneberg A, Nielsen HB, Pelletier E, Renault $\mathrm{P}$, Sicheritz-Ponten T, Turner $\mathrm{K}$, Zhu $\mathrm{H}$, Yu C, Li S, Jian M, Zhou Y, Li Y, Zhang X, Li S, Qin N, Yang H, Wang J, Brunak S, Doré J, Guarner F, Kristiansen K, Pedersen O, Parkhill J, Weissenbach J, Bork P, Ehrlich SD, Wang J et al (2010) A human gut microbial gene catalogue established by metagenomic sequencing. Nature. https://doi. org/10.1038/nature08821

13. Caussy C, Tripathi A, Humphrey $G$, Bassirian $S$ Singh S, Faulkner C, Bettencourt R, Rizo E, Richards L, Xu ZZ, Downes MR, Evans RM, Brenner DA, Sirlin CB, Knight R, Loomba R (2019) $A$ gut microbiome signature for cirrhosis due to nonalcoholic fatty liver disease. Nat Commun. https://doi.org/10.1038/s41467-019-09455-9

14. Koliada A, Syzenko G, Moseiko V, Budovska L, Puchkov K, Perederiy V, Gavalko Y, Dorofeyev A, Romanenko M, Tkach S, Sineok L, Lushchak O, Vaiserman A (2017) Association between body mass index and firmicutes/bacteroidetes ratio in an adult Ukrainian population. BMC Microbiol. https://doi.org/10.1186/s12866-017-1027-1

15. Schwimmer JB, Johnson JS, Angeles JE, Behling C, Belt PH, Borecki I, Bross C, Durelle J, Goyal NP, Hamilton G, Holtz ML, Lavine JE, Mitreva $M$, Newton KP, Pan A, Simpson PM, Sirlin CB, Sodergren E, Tyagi R, Yates KP, Weinstock G, Salzman NH (2019) Microbiome signatures associated with steatohepatitis and moderate to severe fibrosis in children with nonalcoholic fatty liver disease. Gastroenterology. https://doi.org/10. 1053/j.gastro.2019.06.028

16. Kolodziejczyk AA, Zheng D, Elinav E (2019) Diet-microbiota interactions and personalized nutrition. Nat Rev Microbiol. https://doi.org/10. 1038/s41579-019-0256-8

17. Levy M, Kolodziejczyk AA, Thaiss CA, Elinav E (2017) Dysbiosis and the immune system. Nat Rev Immunol 17:219-232

18. Zaneveld JR, McMinds R, Thurber RV (2017) Stress and stability: applying the Anna Karenina principle 
to animal microbiomes. Nat Microbiol. https://doi. org/10.1038/nmicrobiol.2017.121

19. Chiang JYL (2009) Bile acids: regulation of synthesis. J Lipid Res 50:1955-1966. https://doi. org/10.1194/jlr.R900010-JLR200

20. Wahlström A, Sayin SI, Marschall HU, Bäckhed F (2016) Intestinal crosstalk between bile acids and microbiota and its impact on host metabolism. Cell Metab 24:41-50

21. Begley M, Gahan CGM, Hill C (2005) The interaction between bacteria and bile. FEMS Microbiol Rev 29:625-651

22. Modica S, Petruzzelli M, Bellafante E, Murzilli $S$, Salvatore L, Celli N, Di Tullio G, Palasciano G, Moustafa T, Halilbasic E, Trauner M, Moschetta A (2012) Selective activation of nuclear bile acid receptor FXR in the intestine protects mice against cholestasis. Gastroenterology. https://doi.org/10. 1053/j.gastro.2011.10.028

23. Henao-Mejia J, Elinav E, Jin C, Hao L, Mehal WZ, Strowig T, Thaiss CA, Kau AL, Eisenbarth SC, Jurczak MJ, Camporez JP, Shulman Gl, Gordon Jl, Hoffman HM, Flavell RA (2012) Inflammasomemediated dysbiosis regulates progression of NAFLD and obesity. Nature 482:179-185. https:// doi.org/10.1038/nature10809

24. Balakrishnan A, Polli JE (2006) Apical sodium dependent bile acid transporter (ASBT, SLC10A2): a potential prodrug target. Mol Pharmacol 3(3):223-230

25. Fang S, Suh JM, Reilly SM, Yu E, Osborn O, Lackey D, Yoshihara E, Perino A, Jacinto $S$, Lukasheva $Y$, Atkins AR, Khvat A, Schnabl B, Yu RT, Brenner DA, Coulter S, Liddle C, Schoonjans K, Olefsky JM, Saltiel AR, Downes M, Evans RM (2015) Intestinal FXR agonism promotes adipose tissue browning and reduces obesity and insulin resistance. Nat Med. https://doi.org/10.1038/nm.3760

26. Jiang C, Xie C, Li F, Zhang L, Nichols RG, Krausz KW Cai J, Qi Y, Fang ZZ, Takahashi S, Tanaka N, Desai D, Amin SG, Albert I, Patterson AD, Gonzalez FJ (2015) Intestinal farnesoid $X$ receptor signaling promotes nonalcoholic fatty liver disease. J Clin Invest. https://doi.org/10.1172/JCl76738

27. Chow MD, Lee YH, Guo GL (2017) The role of bile acids in nonalcoholic fatty liver disease and nonalcoholic steatohepatitis. Mol Aspects Med 56:34-44. https://doi.org/10.1016/j.mam.2017. 04.004

28. Jiao N, Baker SS, Chapa-Rodriguez A, Liu W, Nugent CA, Tsompana M, Mastrandrea L, Buck MJ, BakerRD, Genco RJ, Zhu R, ZhuL (2018) Suppressed hepatic bile acid signalling despite elevated production of primary and secondary bile acids in NAFLD.Gut. https://doi.org/10.1136/gutjnl-2017314307

29. Evans JM, Morris LS, Marchesi JR (2013) The gut microbiome: the role of a virtual organ in the endocrinology of the host. J Endocrinol 218(3):R37-47. https://doi.org/10.1530/JOE-13 0131

30. Arnold JW, Roach J, Azcarate-Peril MA (2016) Emerging technologies for gut microbiome research. Trends Microbiol 24(11):887-901. https:// doi.org/10.1016/j.tim.2016.06.008

31. Rau M, Rehman A, Dittrich M, Groen AK, Hermanns HM, Seyfried F, Beyersdorf N, Dandekar T, Rosenstiel P, Geier A (2018) Fecal SCFas and SCFA-producing bacteria in gut microbiome of human NAFLD as a putative link to systemic T-cell activation and advanced disease. United European Gastroenterol J. https://doi.org/10.1177/ 2050640618804444
32. Gill PA, van Zelm MC, Muir JG, Gibson PR (2018) Review article: short chain fatty acids as potential therapeutic agents in human gastrointestinal and inflammatory disorders. Aliment Pharmacol Ther 48(1):15-34. https://doi.org/10.1111/apt.14689

33. Kimura I, Ozawa K, Inoue D, Imamura T, Kimura K, Maeda T, Terasawa K, Kashihara D, Hirano K, Tani T, Takahashi T, Miyauchi S, Shioi G, Inoue H, Tsujimoto G (2013) The gut microbiota suppresses insulin-mediated fat accumulation via the shortchain fatty acid receptor GPR43. Nat Commun. https://doi.org/10.1038/ncomms2852

34. Descamps HC, Herrmann B, Wiredu D, Thaiss CA (2019) The path toward using microbial metabolites as therapies. EBioMedicine 44:747-754 https://doi.org/10.1016/j.ebiom.2019.05.063

35. Thaiss CA, Itav S, Rothschild D, Meijer MT, Levy M, Moresi C, Dohnalová L, Braverman S, Rozin S, Malitsky S, Dori-Bachash M, Kuperman Y, Biton I, Gertler A, Harmelin A, Shapiro H, Halpern Z, Aharoni A, Segal E, Elinav E (2016) Persistent microbiome alterations modulate the rate of post-dieting weight regain. Nature 540:544-551. https://doi.org/10.1038/nature20796

36. Yuan J, Chen C, Cui J, Lu J, Yan C, Wei X, Zhao X Li N, Li S, Xue G, Cheng W, Li B, Li H, Lin W, Tian C, Zhao J, Han J, An D, Zhang Q, Wei H, Zheng M, MaX, Li W, Chen X, Zhang Z, Zeng H, Ying S, Wu J, Yang R, Liu D (2019) Fatty liver disease caused by highalcohol-producing klebsiella pneumoniae. Cell Metab 30:675-688.e7. https://doi.org/10.1016/j. cmet.2019.08.018

37. Thaiss CA, Levy M, Grosheva I, Zheng D, Soffer E, Blacher E, Braverman S, Tengeler AC, Barak O, Elazar M, Ben-Zeev R, Lehavi-Regev D, Katz MN, Pevsner-Fischer M, Gertler A, Halpern Z, Harmelin A, Aamar S, Serradas P, Grosfeld A, Shapiro H, Geiger B, Elinav E (2018) Hyperglycemia drives intestinal barrier dysfunction and risk for enteric infection.Science 359:1376-1383. https://doi.org/ 10.1126/science.aar3318

38. Sorribas M, Jakob MO, Yilmaz B, Li H, Stutz D, Noser Y, de Gottardi A, Moghadamrad S, Hassan M, Albillos A, Francés R, Juanola O, Spadoni I, Rescigno M, Wiest R (2019) FxR-modulates the gutvascular barrier by regulating the entry sites for bacterial translocation in experimental cirrhosis. J Hepatol. https://doi.org/10.1016/j.jhep.2019.06. 017

39. Depommier C, Everard A, Druart C, Plovier H, Van Hul M, Vieira-Silva S, Falony G, Raes J, Maiter D, Delzenne NM, de Barsy M, Loumaye A, Hermans MP, Thissen JP, de Vos WM, Cani PD (2019) Supplementation withakkermansiamuciniphilain overweight and obese human volunteers: a proof-of-concept exploratory study. Nat Med 25(7):1096-1103. https://doi.org/10.1038/s41591-019-0495-2

40. Shogbesan $O$, Poudel DR, Victor $S$, Jehangir $A$, Fadahunsi O, Shogbesan G, Donato A (2018) A systematic review of the efficacy and safety of fecal microbiota transplant for clostridium difficile infection in Immunocompromised patients. Can J Gastroenterol Hepatol 2018:1394379. https://doi. org/10.1155/2018/1394379

41. Schneider KM, Bieghs V, Heymann F, Hu W, Dreymueller D, Liao L, Frissen M, Ludwig A, Gassler N, Pabst O, Latz E, Sellge G, Penders J, Tacke $F$ Trautwein C (2015) CX3CR1 is a gatekeeper for intestinal barrier integrity in mice: limiting steatohepatitis by maintaining intestinal homeostasis. Hepatology 62:1405-1416. https://doi.org/10. 1002/hep.27982

42. Bajaj JS, Salzman NH, Acharya C, Sterling RK, White MB, Gavis EA, Fagan A, Hayward M, Holtz ML,
Matherly S, Lee H, Osman M, Siddiqui MS, Fuchs M, Puri P, Sikaroodi M, Gillevet PM (2019) Fecal microbial transplant capsules are safe in hepatic encephalopathy: a phase 1, randomized, placebocontrolled trial. Hepatology. https://doi.org/10. 1002/hep.30690

43. Parnell JA, Raman M, Rioux KP, Reimer RA (2012) The potential role of prebiotic fibre for treatment and management of non-alcoholic fatty liver disease and associated obesity and insulin resistance. Liver Int 32(5):701-711. https://doi. org/10.1111/j.1478-3231.2011.02730.x

44. Carlson JL, Erickson JM, Lloyd BB, Slavin JL (2018) Health effects and sources of prebiotic dietary fiber. Curr Dev Nutr. https://doi.org/10.1093/cdn/ nzy005

45. Macfarlane S, Macfarlane GT, Cummings JH (2006) Review article: prebiotics in the gastrointestinal tract. Aliment Pharmacol Ther 24(5):701-714

46. Tarantino G, Finelli C (2015) Systematic review on intervention with prebiotics/probiotics in patients with obesity-related nonalcoholic fatty liver disease. Future Microbiol 10(5):889-902. https://doi.org/10.2217/fmb.15.13

47. Singh V, Yeoh BS, Chassaing $B$, Xiao $X$, Saha $P$ Aguilera Olvera R, Lapek JD, Zhang L, Wang WB, Hao S, Flythe MD, Gonzalez DJ, Cani PD, ConejoGarcia JR, Xiong N, Kennett MJ, Joe B, Patterson AD, Gewirtz AT, Vijay-Kumar M (2018) Dysregulated microbial fermentation of soluble fiber induces cholestatic liver cancer. Cell. https://doi.org/10. 1016/j.cell.2018.09.004

48. Younossi Z, Ratziu V, Loomba R, Rinella M, Anstee QM, Goodman Z, Bedossa P, Geier A, Beckebaum S, Newsome P, Sheridan D, Trotter J, Knapple W, Lawitz E, Kowdley K, Montano-Loza A, Boursier J, Mathurin P, Bugianesi E, Mazzella G, Olveira A, Cortez-Pinto H, Graupera I, Orr D, GluudLL, Dufour J-F, Shapiro D, Campagna J, ZaruL, MacConell L, Shringarpure R, Harrison S, Sanyal A (2019) GS-06-positive results from REGENERATE: a phase 3 international, randomized, placebocontrolled study evaluating obeticholic acid treatment for NASH. J Hepatol. https://doi.org/10. 1016/s0618-8278(19)30006-4

49. Nicholes K, Guillet S, Tomlinson E, Hillan K, Wright B, Frantz GD, Pham TA, Dillard-Telm L, Tsai SP, Stephan JP, Stinson J, Stewart T, French DM (2002)A mouse model of hepatocellular carcinoma:ectopic expression offibroblast growth factor 19 in skeletal muscle of transgenic mice. Am JPathol. https://doi. org/10.1016/S0002-9440(10)61177-7

50. Harrison SA, Rinella ME, Abdelmalek MF, Trotter JF, Paredes AH, Arnold HL, Kugelmas M, Bashir MR, Jaros MJ, Ling L, Rossi SJ, DePaoli AM, Loomba R (2018) NGM282 for treatment of non-alcoholic steatohepatitis: a multicentre, randomised, double-blind, placebo-controlled, phase 2 trial. Lancet. https://doi.org/10.1016/S0140-6736(18)30474-4 